Research Paper

\title{
Antitumor Effect of Embryonic Stem Cells in a Non-Small Cell Lung Cancer Model: Antitumor Factors and Immune Responses
}

Wei Dong ${ }^{1,2^{*}}$, Chen Qiu ${ }^{1^{*}}$, Hongchang Shen ${ }^{1}$, Qi Liu ${ }^{1}$, and Jiajun Du ${ }^{1,2^{\circledR}}$

1. Institute of Oncology, Shandong Provincial Hospital affiliated to Shandong University, Shandong University, 324 Jingwu Road, Jinan, 250021 P.R. China;

2. Department of Thoracic Surgery, Shandong Provincial Hospital affiliated to Shandong University, Shandong University, 324 Jingwu Road, Jinan, 250021 P.R. China.

* contributed equally to this work.

$\triangle$ Corresponding author: Jiajun Du, Institute of Oncology \& Department of Thoracic Surgery, Shandong Provincial Hospital affiliated to Shandong University, Shandong University, 324 Jingwu Road, Jinan, 250021 P.R. China, E-Mail: dujiajun@sdu.edu.cn Tel.: 86-531-6877-7100; Fax: 86-531-6877-7100.

(c) Ivyspring International Publisher. This is an open-access article distributed under the terms of the Creative Commons License (http://creativecommons.org/ licenses/by-nc-nd/3.0/). Reproduction is permitted for personal, noncommercial use, provided that the article is in whole, unmodified, and properly cited.

Received: 2013.04.22; Accepted: 2013.07.23; Published: 2013.08.09

\begin{abstract}
Research in recent years has revealed that embryonic stem cells (ESCs) could generate obvious antitumor effects in both vitro and vivo. In vitro, ESCs could secrete soluble factors that are capable of blocking cancer cells proliferation, moreover, embryonic microenvironments could effectively inhibit tumorigenesis and metastasis; while in vivo, administration of ESCs in tumor-bearing mice could generate significant antitumor effects by indirectly activating the antitumor immune system. In this study, non-small cell lung cancer cells (Lewis Lung Carcinoma cells, LLCs) and ESCs were co-injected together into mice, after that subcutaneous tumor growth was monitored, cellular and humoral immune responses were detected, and different control groups were set to compare the results in different conditions. Our results suggested that compared to be injected alone, ESCs co-injected with cancer cells could inhibit cancer cell growth more efficiently in vivo, with more $\mathrm{CD} 8+$ lymphocytes generated in both peripheral circulation and spleen, and with higher serum anticancer cytokine level (interleukin (IL)-2 and interferon (IFN)- $\mathrm{Y}$ ). We conclude that the boosted antitumor effects induced by ESCs and cancer cells co-injection may be both the effects of antitumor factors secreted by ESCs and immune responses induced by ESCs in vivo.
\end{abstract}

Key words: embryonic stem cells; immunotherapy; antitumor immunity; antitumor factors.

\section{Introduction}

Since Lobstein and Recamier put forward the hypothesis that neoplasm originates from embryo cells, investigators made great efforts in the comparative study between neoplasm formation and embryonic development in the last few decades [1]. They found that cancer cells and stem cells have some common biological properties, including unlimited proliferation, the ability of migration and invasion, and they have some similar gene products and signal transduction pathways [2-5]. Moreover, cancer cells and ESCs share some cell surface markers and antigens that not presented by adult tissues [6-8]. On the other hand, ESCs were applied to generate antitumor effects in both vitro and vivo. For example, ESCs could produce soluble factors arresting or slowing population growth of cancer cell lines [9], and embryonic microenvironments have the potential to reprogram cancer cells into a less invasive pheno- 
type and help preventing tumorigenesis and metastasis $[10,11]$. In vivo studies, mice vaccinated with ESCs could induce specific antitumor responses effectively protecting them from tumor formation and development [12]; even in mice with minor and heavy tumor load, administration of ESCs could generate effective antitumor immunity, which obviously improved the immune dysfunction and inhibit tumor growth [13].

So far, it is not hard to conclude that ESCs have double hit against cancer: direct inhibition by antitumor factors and indirect suppression by activating the body immune system. We hypothesized that these two aspects can give a combined effect when ESCs can get direct contact with cancer cells in vivo. In this study, mice were co-injected with ESCs and cancer cells simultaneously, and two different control groups were set. As expected, the results showed that ESCs co-injected with cancer cells could induce stronger anticancer immune responses in vivo, with more CD8+ lymphocytes generated and higher anticancer cytokine level (IL-2 and IFN- $\gamma$ ) compared to be injected alone.

\section{Results and Discussion}

\section{I Inhibition of tumor growth by administra- tion of ESCs}

Lewis Lung Carcinoma cell is a well-established carcinoma cell line in mice [14], and $1 \times 10^{6}$ cells were enough to induce subcutaneous tumor masses and eventual death. The tumor formation time and tumor growth of all groups of mice were closely monitored after LLCs administration (Fig.1A, C), and the tumor weight was recorded at the endpoint of experiment (Fig.1B). The results suggested that mice with ESCs injection (LLC+ESC and LLC+contra ESC group) generated obvious antitumor responses and rejected tumor masses from formation and development effectively compared to the LLC+MEF (Mouse Embryonic Fibroblast cells) group $(p<0.05)$; moreover, mice in LLC+ESC group obtained stronger antitumor effects in inhibition of tumor masses compared with mice in LLC+contra ESC group $(p<0.05)$, which means more significant antitumor effect could be induced when ESCs got direct contact with tumor cells in vivo.

A

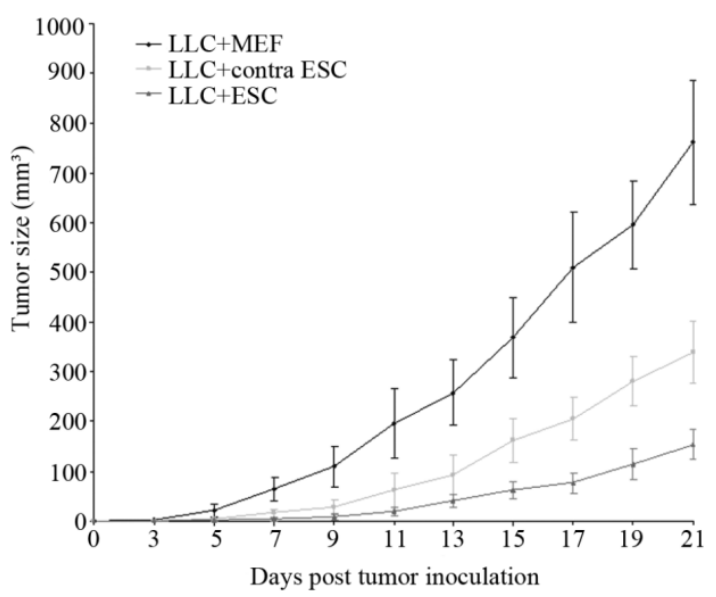

B
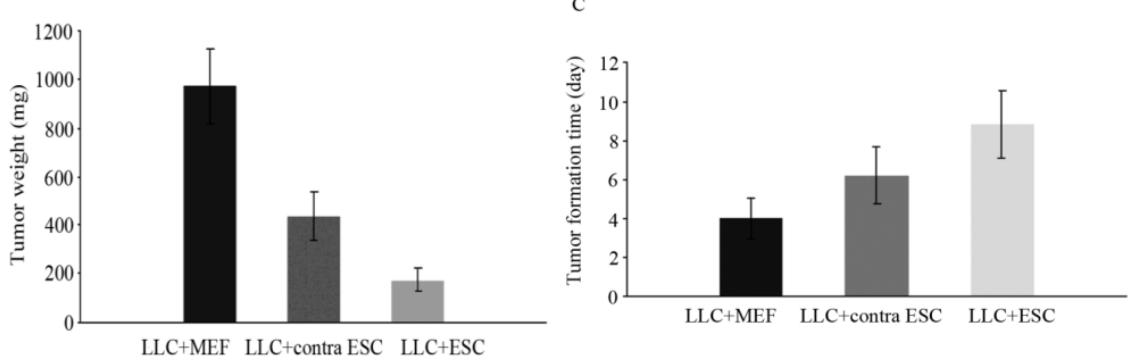

Fig I. (A) Tumor size of all groups was measured and tumor volumes were calculated after LLCs inoculation. The results indicated statistical difference in tumor size between mice injected with ESCs (LLC+contra ESC and LLC+ESC) and MEFs (LLC+MEF) from day 5 on $(p<0.05)$, and between groups in which ESCs could get direct contact with cancer cells (LLC+ESC) or not (LLC+contra ESC) ( $p<0.05$ from day 9 on). (B) All mice were sacrificed under anesthesia and tumor weight was detected at the end of the experiment. The results suggested significant difference in tumor weight between ESCs (LLC+contra ESC and LLC+ESC) and MEFs (LLC+MEF) groups, and between the LLC+ESC and LLC+contra ESC group ( $p<0.05)$. (C) Tumor formation time of all groups. The results suggested the tumor formation time was statistically different between the experimental (LLC+ESC) and control groups (LLC+MEF and LLC+contra ESC) $(p<0.05)$. Error bars denote SD. 


\subsection{Increased proliferation of lymphocytes and secretion of cytokines}

To explore the specific changes in immune system, we examined the proliferation of lymphocytes in the peripheral circulation and spleen of all mice induced by ESCs administration by flow cytometry and IHC in our study (Fig. 2 and 3). We found statistically significant proliferation of both $\mathrm{CD}^{+}+$and CD8+ lymphocytes in both blood and spleen of mice injected by ESCs (LLC+ESC and LLC+contra ESC group) compared to the LLC+MEF group $(p<0.05)$. And compared to the LLC+contra ESC group, mice in LLC+ESC group generated more CD8+ lymphocytes in both peripheral circulation and spleen $(p<0.05)$.

To further study the changes in cellular immune responses induced by ESCs administration, we detected the level of immune important cytokines in serum of all mice. The concentration of IL-4, IL-2 and IFN- $\gamma$ was detected by ELISA kits (Fig. 4), and the results suggested that mice in LLC+ESC and LLC+contra ESC group had significantly increased level of IL-2 and IFN- $\gamma(p<0.05)$, but no obvious change in the serum level of IL-4. Furthermore, compared with the LLC+contra ESC group, mice in LLC+ESC group had a statistically higher level of IFN- $\gamma$ in serum $(p<0.05)$.

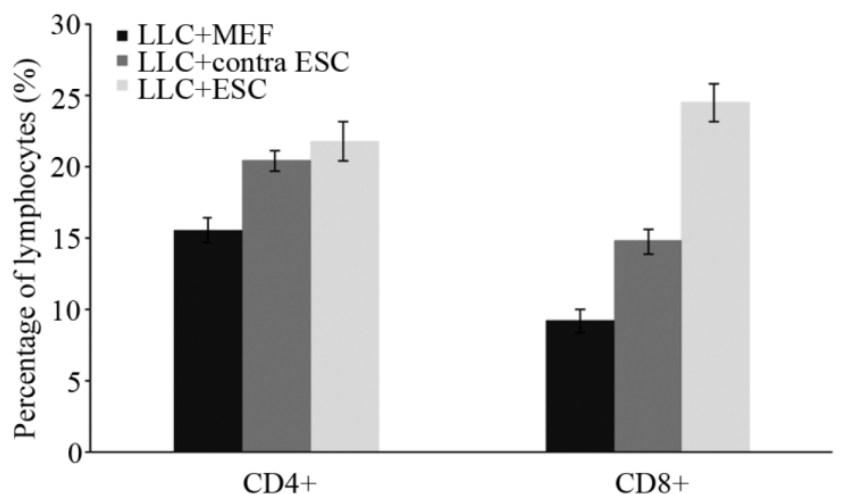

Fig 2. There was statistical difference in the percentage of lymphocytes between mice injected with ESCs (LLC+contra ESC and LLC+ESC) and MEFs (LLC+MEF) $(p<0.05)$, and significant difference in the percentage CD8+ lymphocytes between the LLC+ESC and LLC+contra ESC group ( $p$ $<0.05)$.

\subsection{Increased cell apoptosis in tumor tissues}

When the mice were sacrificed at the endpoint of the study, we detected the cell apoptosis in the tumor tissues by TUNEL kits (Fig. 5). We found more cell apoptosis in the LLC+ESC and LLC+contra ESC group compared to the LLC+MEF group $(p<0.05)$. Moreover, the apoptosis level of tumor cells in mice from LLC+ESC group was statistically different from mice in LLC+contra ESC group $(p<0.05)$.

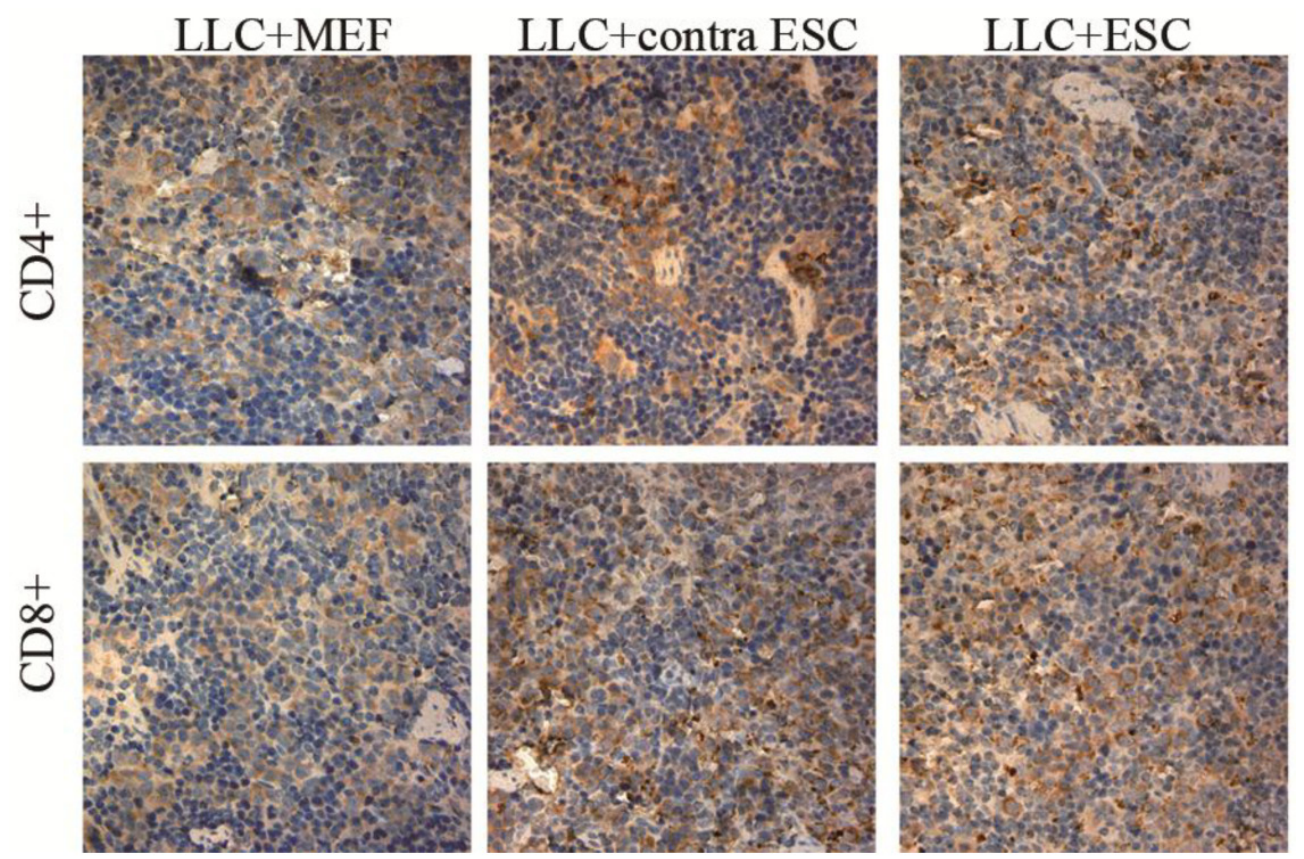

Fig 3. The mean optical density for CD4 positive $T$ lymphocytes was $0.17 \pm 0.021$ for LLC+MEF group, $0.25 \pm 0.019$ for LLC+contra ESC group and $0.27 \pm 0.032$ for LLC+ESC group. The mean optical density for CD8 positive T lymphocytes was $0.16 \pm 0.017$ for LLC+MEF group, $0.27 \pm 0.030$ for LLC+contra ESC group and $0.34 \pm 0.037$ for LLC+ESC group. The results indicated statistical difference between mice injected with ESCs (LLC+contra ESC and LLC+ESC) and MEFs (LLC+MEF) in the CD4 and CD8 positive T lymphocytes in spleens $(p<0.05)$. Moreover, significant difference was detected between the LLC+ESC and LLC+contra ESC group in CD8 positive T lymphocytes $(p<0.05)$. 


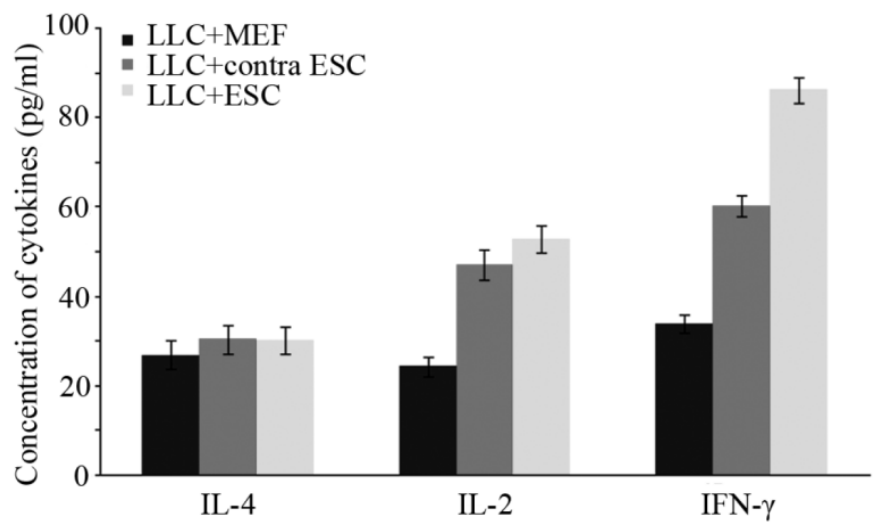

Fig 4. There was statistical difference in the serum level of IL-2 and IFN-y between mice injected with ESCs (LLC+contra ESC and LLC+ESC) and MEFs (LLC+MEF) $(p<0.05)$, but no obvious change in the serum level of IL-4. Furthermore, compared with the LLC+contra ESC group, mice in LLC+ESC group had a statistically higher level of IFN-y in serum $(p<0.05)$.

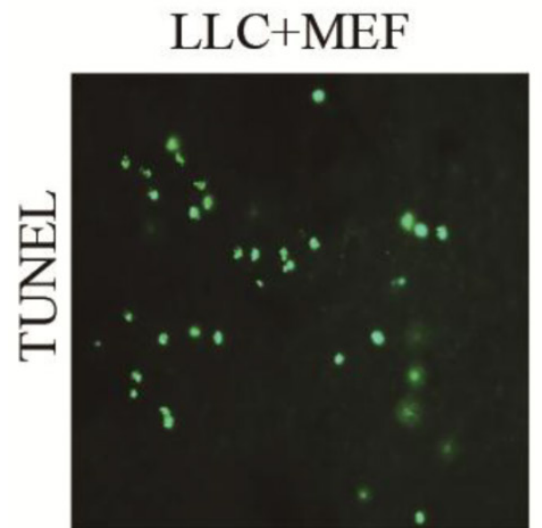

LLC+contra ESC

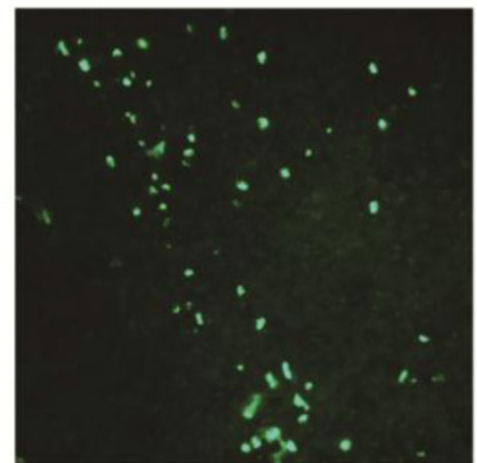

$\mathrm{LLC}+\mathrm{ESC}$

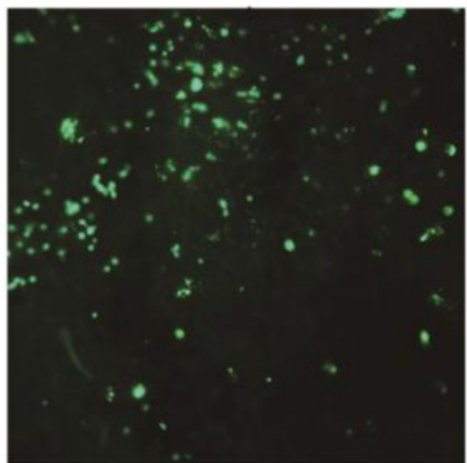

Fig 5. The mean optical density for TUNEL cell apoptosis was $0.041 \pm 0.0034$ for LLC+MEF group, $0.075 \pm 0.0040$ for LLC+contra ESC group and $0.092 \pm 0.0047$ for LLC+ESC group. The results suggested statistical difference between mice injected with ESCs (LLC+contra ESC and LLC+ESC) and MEFs (LLC+MEF) $(p<0.05)$, moreover, compared with the LLC+contra ESC group, mice in LLC+ESC group had a statistically higher level of cell apoptosis $(p$ $<0.05)$.

\subsection{Discussion}

Lung cancer is known to be the most commonly diagnosed cancer as well as the leading cause of cancer related death around the world [15]. The five-year survival rate remains less than $15 \%$ despite of improvement in surgery, radiotherapy and chemotherapy. There has been a strong requirement in new effective and better tolerated treatment strategies. Lack of appropriate lymphocytes activation for tumor rejection was thought to be an important reason why immune system failed to eliminate a tumor mass [16, 17]. The mechanisms for cancer cells to escape from immune recognition may be the alteration of proteins involved in antigen processing and presentation, loss of major histocompatibility complex class I expression and presence of immunosuppressive factors. And finally, insufficient activation of antitumor immunity lead to poor protection from tumors [18-20].

Since the first observation that embryonic materials could be used as vaccines to prevent tumor for- mation and development in animal models, there has been accumulative evidence supporting the fact that embryonic/fetal materials could generate effective antitumor responses in vivo [21]. Now it has been well proved that ESCs and their derivatives could generate effective cellular immune response and active CD4+ and CD8+ lymphocytes [22-24]. Because of the antigenic similarities between ESCs and tumor cells, immune responses against ESCs could cross-react with tumor cells, so antitumor immunity in vivo was generated $[12,25]$.

In our study, we found a strong and steady activation of antitumor cellular immune response by ESCs administration, we detected obvious proliferation of lymphocytes (in both peripheral circulation and spleen) and especially a dramatic increase of CD8+ lymphocytes (cytotoxic T lymphocytes) which was believed to consist the main part of antitumor immunity and play an crucial role in tumor rejection [26-28]. Moreover, increase in the serum level of Th1 cytokines (IL-2 and IFN- $\gamma$ ) secreted by lymphocytes 
was also detected, these cytokines played an important role in regulating the antitumor immune responses and differentiation of cytotoxic $\mathrm{T}$ lymphocytes [29-31]. So we concluded that the induced immune responses were not only an increase in quantity of lymphocytes but also an enhancement in cellular immune function, which help to rebuild the host's immunity against cancer [13]. And these antitumor immune responses lead to obvious retardation in the formation and development of tumor masses, including the delayed tumor formation time, inhibited tumor growth in both size and weight, and more cell apoptosis in tumor sections.

However, the more attractive part in this study was that, compared with the LLC+contra ESC group, mice in LLC+ESC group seemed to generate more effective antitumor responses when ESCs could get direct contact with cancer cells in vivo. In these mice, we found more significant inhibition in formation and growth of tumor masses, more effectively activation of lymphocytes proliferation and cytokine secretion, and significant difference in tumor cell apoptosis without obvious clinical signs of autoimmune disease. There are several potential mechanisms that could account for the boosted antitumor response in the LLC+ESC group. We believed that it was a complex result of both direct inhibition effects by ESCs and induced immune responses. As recent studies indicated that stem cells could secrete antitumor factors and inhibit cancer cells proliferation [9, 32, 33], and embryonic microenvironments have an essential role in preventing carcinogenesis by providing signals to inhibit malignant cells proliferation and promote differentiation $[10,11,34]$. On the other hand, the immunogenicity of ESCs played an important part in tumor rejection, which induced cellular immune responses, recruited cytotoxic $\mathrm{T}$ lymphocytes and lead to tumor cells apoptosis as specifically described above.

However, it is still difficult to illustrate a specific mechanism responsible for the rejection of tumors, as it is known to be a complicated one involving lymphocytes and other immune effector cells, soluble factors such as nitric oxide, chemokine ligand and cytokines $[12,35]$. And some issues are still necessary to be explored for further experiments, such as more cell lines and multiple experiment are required to make a general statement, whether the immune responses are tumor specific, what kind of effector cells are involved in the co-injection site of ESCs and cancer cells, etc. But our study may introduce a new dimension on the antitumor application of ESCs and therapeutic strategy for cancer.

\section{Materials and methods}

\section{I Mice and cell lines}

Wild type female C57 BL/6 mice were obtained from the Institute of Laboratory Animal Science, Chinese Academy of Medical Science, 4-6 weeks old on arrival and raised under special pathogen-free conditions for 2 weeks before use. The C57 BL/6 Mouse Embryonic Stem Cells were obtained from Cyagen Bioscience Inc., cultured on plates pre-coated with gelatin solution and $\gamma$-ray irradiated C57 BL/6 Mouse Embryonic Fibroblast cells (MEFs) (Cyagen Bioscience Inc.) as feeder cells, and grew in Mouse Embryonic Stem Cell Growth Medium (Cyagen Bioscience Inc.). The ESCs were in passage 8-10 approximately and pre-irradiated (15Gy/3 fractions) when used as cellular therapy. The Lewis Lung Carcinoma cells were purchased from the Cell Bank of Chinese Academy of Medical Science, cultured in Dulbecco's Modified Eagle's Medium (DMEM, Hyclone) supplemented with 10\% Fetal Bovine Serum (FBS, Hyclone), and were in passage 10-15 when inoculated.

\subsection{Reagent and antibodies}

Most reagents used in cell culture were purchased from Hyclone Biochemical Products Co., Ltd unless specially indicated. The antibodies and reagents used in flow cytometry were obtained from eBioscience (San Diego, CA, USA), and antibodies used in immunohistochemistry were from BioWorld Technology Ltd (Barrie, ON, Canada) and Biosynthesis Biotechnology Co., Ltd (Beijing, China). The ELISA kits were obtained from Jingmei Biotech Co., Ltd (Beijing, China) and one step TUNEL apoptosis assay kits were purchased from Beyotime Biotechnology (Haimen, China).

\subsection{Experiment protocols}

Thirty female C57 BL/ 6 mice were randomly divided into three groups: mice in experimental group (LLC+ESC) $(\mathrm{n}=10)$ received subcutaneous administration of a mixture of $1 \times 106$ LLCs and $1 \times 106$ ESCs in the left axilla; mice in control group $(\mathrm{LLC}+\mathrm{MEF})(\mathrm{n}=10)$ received subcutaneous injection of a mixture of $1 \times 106$ LLCs and $1 \times 106$ MEFs in left axilla; and mice in another control group (LLC+contra ESC) $(n=10)$ received subcutaneous administration of $1 \times 106$ LLCs in left axilla and $1 \times 106$ ESCs in right axilla. Tumor sizes were measured by digital calipers in longitudinal $(L)$ and transverse $(W)$ diameters every other day since tumor cells inoculation and tumor volumes were calculated according to the formula $V=L \times W^{2} / 2$ [36]. The endpoint for this study was set to be three weeks after tumor cells administration, and then all mice were sacrificed under anesthesia and 
tumors were exercised and weighed. During the experiment, all mice were monitored for general health indicators: general behavior, feeding and defecation, body weight, neuromuscular tone, appearance of fur, etc. All animal experiments were carried out using a protocol approved by the Ethics Committee of Provincial Hospital affiliated to Shandong University, Shandong University.

\subsection{Flow cytometry}

Three days after the cell injection, citrate-anticoagulated blood was acquired from venous sinus of the eye orbits in mice from all groups as described [37]. Sampled blood was stained with antibodies for CD3, CD4 and CD8 positive lymphocytes and analyzed on an EPICS XL flow cytometer (Beckman Coulter Corp.), and the results were processed by the WinMDI software (Scripps Institute).

\subsection{ELISA}

Fresh blood was obtained from all mice at the same time and in the same way as previously described, and serum was collected. Quantification of IL-4, IL-2 and IFN- $\gamma$ was performed using commercially available enzyme-linked immunosorbent assay kits in accordance with the instructions and analyzed on a micro-plate reader (Wellscan MK3, Labsystems Dragon).

\subsection{Histology and immunohistochemistry (IHC)}

All mice were sacrificed under anesthesia at the endpoint of the study, and the primary tumors and spleens were excised and fixed in $4 \%$ formaldehyde solution. Tissue samples were embedded in paraffin wax and serially sectioned. Tissue sections were deparaffinized and rehydrated through a graded ethanol series, followed by incubation with 3\% hydrogen peroxide for $15 \mathrm{~min}$. After 15-min rinses in three changes of Phosphate Buffered Saline (PBS), sections were blocked with normal goat serum to suppress nonspecific background staining, and incubated at $4^{\circ} \mathrm{C}$ overnight with diluted (1: 100) CD4 and CD8 antibodies. Then the sections were incubated with biotinylated goat anti-rabbit IgG for $30 \mathrm{~min}$ at room temperature and processed according to the manufacturer's protocol, and finally examined under a Nikon E200 light microscope (Nikon, Tokyo, Japan) equipped with a digital camera system.

\subsection{TUNEL}

The tumor sections were deparaffinized and rehydrated through a graded ethanol series, incubated with protease $\mathrm{K}$ for $25 \mathrm{~min}$ at room temperature for antigen retrieval, and then operated according to the protocol of the TUNEL apoptosis detection kit. The FITC-labeled positive cells were imaged under a fluorescent microscope using 488-nm excitation and 530-nm emission [38]. Quantification of the positive expression of both immunohistochemistry and TUNEL was performed using the mean optical density method as described $[13,39]$.

\subsection{Statistical analysis}

All data were expressed as the mean \pm standard error. Statistical significance between groups was determined by ANOVA test and Student-Newman-Keuls multiple comparisons test after confirming the equality of variance with the Levene test using SPSS 17.0 statistical software (SPSS Inc, Chicago, IL). For all tests, a $p$ value less than 0.05 was considered to be statistically significant.

\section{Acknowledgement}

This study was supported by National natural science foundation of China (81141100), Provincial natural science foundation of Shandong (2010ZRB14180 and 2011ZRB14192) and Provincial science and technology foundation of Shandong (2011GG21819).

\section{Competing Interests}

The authors have declared that no competing interest exists.

\section{References}

1. Murray MJ, Lessey BA. Embryo implantation and tumor metastasis: common pathways of invasion and angiogenesis. Semin Reprod Endocrinol. 1999; 17: 275-90.

2. Pardal R, Clarke MF, Morrison SJ. Applying the principles of stem-cell biology to cancer. Nat Rev Cancer. 2003; 3: 895-902.

3. Cabanillas R, Llorente JL. The Stem Cell Network model: clinical implications in cancer. Eur Arch Otorhinolaryngol. 2009; 266: 161-70.

4. Reya T, Clevers H. Wnt signalling in stem cells and cancer. Nature. 2005; 434: 843-50

5. Li L, Neaves WB. Normal stem cells and cancer stem cells: the niche matters. Cancer Res. 2006; 66: 4553-7.

6. Huang EH, Heidt DG, Li CW, Simeone DM. Cancer stem cells: a new paradigm for understanding tumor progression and therapeutic resistance. Surgery. 2007; 141: 415-9.

7. Okamoto OK. Cancer stem cell genomics: the quest for early markers of malignant progression. Expert Rev Mol Diagn. 2009; 9: 545-54.

8. Rubio D, Garcia-Castro J, Martin MC, de la Fuente R, Cigudosa JC, Lloyd $\mathrm{AC}$, et al. Spontaneous human adult stem cell transformation. Cancer Res. 2005; 65: 3035-9.

9. Giuffrida D, Rogers IM, Nagy A, Calogero AE, Brown TJ, Casper RF. Human embryonic stem cells secrete soluble factors that inhibit cancer cell growth. Cell Prolif. 2009; 42: 788-98.

10. Raof NA, Raja WK, Castracane J, Xie Y. Bioengineering embryonic stem cell microenvironments for exploring inhibitory effects on metastatic breast cancer cells. Biomaterials. 2011; 32: 4130-9.

11. Postovit LM, Margaryan NV, Seftor EA, Kirschmann DA, Lipavsky A, Wheaton WW, et al. Human embryonic stem cell microenvironment suppresses the tumorigenic phenotype of aggressive cancer cells. Proc Natl Acad Sci U S A. 2008; 105: 4329-34.

12. Li Y, Zeng H, Xu RH, Liu B, Li Z. Vaccination with human pluripotent stem cells generates a broad spectrum of immunological and clinical responses against colon cancer. Stem Cells. 2009; 27: 3103-11.

13. Dong W, Du J, Shen H, Gao D, Li Z, Wang G, et al. Administration of embryonic stem cells generates effective antitumor immunity in mice 
with minor and heavy tumor load. Cancer Immunol Immunother. 2010; 59: 1697-705.

14. Mayo JG. Biologic characterization of the subcutaneously implanted Lewis lung tumor. Cancer Chemother Rep 2. 1972; 3: 325-30.

15. Jemal A, Bray F, Center MM, Ferlay J, Ward E, Forman D. Global cancer statistics. CA Cancer J Clin. 2011; 61: 69-90.

16. Sioud M. Does our current understanding of immune tolerance, autoimmunity, and immunosuppressive mechanisms facilitate the design of efficient cancer vaccines? Scand J Immunol. 2009; 70: 516-25.

17. Grivennikov SI, Greten FR, Karin M. Immunity, inflammation, and cancer. Cell. 2010; 140: 883-99.

18. Xiong G, Husseiny MI, Song L, Erdreich-Epstein A, Shackleford GM, Seeger RC, et al. Novel cancer vaccine based on genes of Salmonella pathogenicity island 2 . Int J Cancer. 2010; 126: 2622-34.

19. Johnsen A, France J, Sy MS, Harding CV. Down-regulation of the transporter for antigen presentation, proteasome subunits, and class I major histocompatibility complex in tumor cell lines. Cancer Res. 1998; 58: 3660-7.

20. Khong HT, Restifo NP. Natural selection of tumor variants in the generation of "tumor escape" phenotypes. Nat Immunol. 2002; 3: 999-1005.

21. Brewer BG, Mitchell RA, Harandi A, Eaton JW. Embryonic vaccines against cancer: an early history. Exp Mol Pathol. 2009; 86: 192-7.

22. Drukker M, Katchman H, Katz G, Even-Tov Friedman S, Shezen E, Hornstein E, et al. Human embryonic stem cells and their differentiated derivatives are less susceptible to immune rejection than adult cells. Stem Cells. 2006; 24: 221-9.

23. Boyd AS, Higashi Y, Wood KJ. Transplanting stem cells: potential targets for immune attack. Modulating the immune response against embryonic stem cell transplantation. Adv Drug Deliv Rev. 2005; 57: 1944-69.

24. Fairchild PJ, Cartland S, Nolan KF, Waldmann H. Embryonic stem cells and the challenge of transplantation tolerance. Trends Immunol. 2004; 25: 465-70.

25. Mocan T, Iancu C. Effective colon cancer prophylaxis in mice using embryonic stem cells and carbon nanotubes. Int J Nanomedicine. 2011; 6: 1945-54.

26. Begley J, Ribas A. Targeted therapies to improve tumor immunotherapy. Clin Cancer Res. 2008; 14: 4385-91.

27. Klebanoff CA, Gattinoni L, Restifo NP. CD8+ T-cell memory in tumor immunology and immunotherapy. Immunol Rev. 2006; 211: 214-24.

28. Antony PA, Piccirillo CA, Akpinarli A, Finkelstein SE, Speiss PJ, Surman $\mathrm{DR}$, et al. CD8+ $\mathrm{T}$ cell immunity against a tumor/self-antigen is augmented by CD4+ T helper cells and hindered by naturally occurring T regulatory cells. J Immunol. 2005; 174: 2591-601.

29. Yamamoto M, Kamigaki T, Yamashita K, Hori Y, Hasegawa H, Kuroda D, et al. Enhancement of anti-tumor immunity by high levels of Th1 and Th17 with a combination of dendritic cell fusion hybrids and regulatory T cell depletion in pancreatic cancer. Oncol Rep. 2009; 22: 337-43.

30. Apte SH, Groves P, Olver S, Baz A, Doolan DL, Kelso A, et al. IFN-gamma inhibits IL-4-induced type 2 cytokine expression by CD8 T cells in vivo and modulates the anti-tumor response. J Immunol. 2010; 185: 998-1004.

31. Nagai H, Miyaki D, Matsui T, Kanayama M, Higami K, Momiyama K, et al. Th1/Th2 balance: an important indicator of efficacy for intra-arterial chemotherapy. Cancer Chemother Pharmacol. 2008; 62: 959-63.

32. Qiao L, Xu Z, Zhao T, Zhao Z, Shi M, Zhao RC, et al. Suppression of tumorigenesis by human mesenchymal stem cells in a hepatoma model. Cell Res. 2008; 18: 500-7.

33. Ramasamy R, Lam EW, Soeiro I, Tisato V, Bonnet D, Dazzi F. Mesenchymal stem cells inhibit proliferation and apoptosis of tumor cells: impact on in vivo tumor growth. Leukemia. 2007; 21: 304-10.

34. Postovit LM, Seftor EA, Seftor RE, Hendrix MJ. A three-dimensional model to study the epigenetic effects induced by the microenvironment of human embryonic stem cells. Stem Cells. 2006; 24: 501-5.

35. Klopp AH, Gupta A, Spaeth E, Andreeff M, Marini F, 3rd. Concise review: Dissecting a discrepancy in the literature: do mesenchymal stem cells support or suppress tumor growth? Stem Cells. 2011; 29: 11-9.

36. Rosato A, Zoso A, Dalla Santa S, Milan G, Del Bianco P, De Salvo GL, et al. Predicting tumor outcome following cancer vaccination by monitoring quantitative and qualitative CD8+ T cell parameters. J Immunol. 2006; 176: 1999-2006.

37. Fernandez I, Pena A, Del Teso N, Perez V, Rodriguez-Cuesta J. Clinical biochemistry parameters in C57BL/6J mice after blood collection from the submandibular vein and retroorbital plexus. J Am Assoc Lab Anim Sci. 2010; 49: 202-6.

38. Wang Z, Tang $X$, Li Y, Leu C, Guo L, Zheng $X$, et al. 20-Hydroxyeicosatetraenoic acid inhibits the apoptotic responses in pulmonary artery smooth muscle cells. Eur J Pharmacol. 2008; 588: 9-17.
39. Chen BA, Jin N, Wang J, Ding J, Gao C, Cheng J, et al. The effect of magnetic nanoparticles of $\mathrm{Fe}(3) \mathrm{O}(4)$ on immune function in normal ICR mice. Int J Nanomedicine. 2010; 5: 593-9. 